PROCEEDINGS OF THE

AMERICAN MATHEMATICAL SOCIETY

Volume 135, Number 3, March 2007, Pages 873-881

S 0002-9939(06)08579-0

Article electronically published on October 19, 2006

\title{
NONRIGIDITY OF HYPERBOLIC SURFACES LAMINATIONS
}

\author{
BERTRAND DEROIN
}

(Communicated by Linda Keen)

\begin{abstract}
In this paper we prove infinite dimensionality of the Teichmüller space of a hyperbolic Riemann surface lamination of a compact space having a simply connected leaf.
\end{abstract}

\section{INTRODUCTION}

A Riemann surfaces lamination $\mathcal{L}$ of a topological space $X$ is an atlas of homeomorphisms from open sets covering $X$ to the product of the unit disc by a topological space, called transverse space, such that the changes of coordinates preserve the local fibration by discs and are holomorphic along the fibers. D. Sullivan discovered some interesting relations between $C^{1}$-conjugacy classes of dynamical systems of dimension 1 and the space of conformal structures on a lamination by smooth surface. In $[\mathrm{Su}$, he made the systematic study of the Teichmüller space of a Riemann surface lamination. It is defined as the space of all continuous conformal structures along the leaves (transversally continuous Beltrami coefficients), modulo the group of quasi-conformal isotopies tangent to the leaves. It is then proved that if the leaves are hyperbolic and the space is compact, there is a "laminated" Bers embedding theorem. Namely, the natural topology on the Teichmüller space is Hausdorff, and it is biholomorphic to a nonempty open subset of the Banach space of holomorphic quadratic differentials along the leaves and continuous on the total space.

In this paper we prove the infinite dimensionality of Teichmüller space for a wide class of Riemann surface laminations of a compact space whose leaves are hyperbolic, by producing an infinite dimensional space of continuous holomorphic quadratic differentials.

Theorem 1.1. Let $\mathcal{L}$ be a Riemann surfaces lamination of a compact space $X$ with hyperbolic leaves and suppose that $\mathcal{L}$ possesses a simply connected leaf. Then the Banach space of continuous holomorphic quadratic differentials is infinite dimensional.

In fact, we prove the theorem under a weaker hypothesis. We prove that there exists a universal constant $C$ such that if the hyperbolic Riemann surfaces lamination has a leaf $L$ and a point $x$ on $L$ such that $L$ is not transversally isolated and

Received by the editors October 12, 2004.

2000 Mathematics Subject Classification. Primary 57R30; Secondary 30Fxx.

Key words and phrases. Teichmüller theory, Riemann surface lamination.

The author acknowledges support from the Swiss National Science Foundation.

(C)2006 American Mathematical Society Reverts to public domain 28 years from publication 
the radius of injectivity at $x$ for the hyperbolic metric in $L$ is greater than $C$, then the theorem is true.

In his famous memoir $[\mathrm{Po}, \mathrm{H}$. Poincaré proved the existence of nontrivial meromorphic quadratic differentials on a compact Riemann surface of genus greater than 2. Let us recall his original method: A compact Riemann surface of genus at least 2 is the quotient of the unit disc $\mathbf{D}$ by a discrete cocompact group $\Gamma$ of biholomorphisms. Let $f$ be a meromorphic function on the disc which is bounded at infinity and consider the following fuchsian series:

$$
\sigma=\sum_{\gamma \in \Gamma} \gamma^{*}\left(f(z) d z^{2}\right)
$$

Modulo convergence it defines a meromorphic quadratic differential having poles at the projection of the poles of $f$ on $\Sigma$. H. Poincaré observed that the series $\sigma$ converges: if $z$ is a point of the disc which is not in an orbit of a pole of $f$, the module of $f(\gamma(z)) \gamma^{\prime}(z)^{2}$ is approximately the euclidian area of the image by $\gamma$ of a fixed small disc centered at $z$. So since $\Gamma$ is discrete and the disc has finite euclidian area, the series $\sigma$ converges on compact subsets of the disc.

In Gh, É. Ghys generalizes this method on a hyperbolic Riemann surface lamination $\mathcal{L}$ of a compact space having a total transversal $\mathcal{T}$. This enables him to construct a continuous meromorphic quadratic differential with given poles on $\mathcal{T}$. He considers on each leaf of the lamination a fuchsian series à la Poincaré. Using the uniformization theory of A. Candel $\mathrm{Ca}$ and A. Verjovsky $\mathrm{Ve}$, he proves that the meromorphic quadratic differential is continuous on the total space, if the family of poles is continuous. In this paper we essentially follow the same method, the originality being that we construct holomorphic quadratic differentials. The existence of a total transversal is then a consequence of our construction. The difficult part is to show that the fuchsian series $\sigma$ does not vanish identically. For instance, in Poincaré's proof, the dimension of the space of quadratic differentials on $\Sigma$ is $3 g-3$; there exist many polynomials $f$ such that the series $\sigma$ vanishes identically.

The paper is organized as follows. In Section 2 we define the fuchsian series $\sigma(q)$ associated to a bounded quadratic differential $q$ on the hyperbolic disc, whose support is $r$-separated. For that we extend each value $q_{t}$ of $q$ by the holomorphic quadratic differential $\tilde{q_{t}}$ of minimizing $L^{1}$ norm and we define

$$
\sigma(q):=\sum_{t \in \mathbf{D}} \tilde{q}_{t}
$$

Uniform convergence of $\sigma(q)$ on compact subsets of the disc is proved in Lemma2.2. In Section 3 we present some continuity properties of $\sigma$. We introduce a topology on the space $\mathcal{D} \mathcal{Q}$ of quadratic differential on the disc bounded by $C>0$ and whose support is $r$-separated, for given constants $r, C>0$. Then we consider the map $\sigma: \mathcal{D Q} \rightarrow H Q(B)$, where $H Q(B)$ is the Banach space of bounded holomorphic quadratic differential on the unit hyperbolic ball $B$, and we prove that it is continuous. In Section 4, we construct fuchsian series on a hyperbolic Riemann surface lamination of a compact space. Each leaf intersects a transversal on a separated subset for the hyperbolic metric. If $q$ is a continuous quadratic differential on the transversal we define on the universal cover of each leaf the fuchsian series $\sigma(q)$. These series are invariant by the fundamental group of the leaves and produce a holomorphic quadratic differential on $X$. We prove the continuity of $\sigma(q)$ in Lemma 4.2 if $q$ vanishes on the boundary of the transversal in the transverse topology. We 
use the unifomization theory of A. Candel and A. Verjovsky. This is the crucial part of the paper. We end in Section 5 with the proof of the theorem.

I thank Étienne Ghys and Alexei Glutsyuk for many helpful conversations.

\section{FUCHSIAN SERIES}

Let $\mathbf{D}=\{z \in \mathbf{C} /|z|<1\}$ be the unit disc and $\tau$ a holomorphic quadratic differential on $\mathbf{D}$. The $L^{1}$-norm of $\tau$ is the volume of $(\mathbf{D},|\tau|)$ where $|\tau|$ is considered as a singular metric on $\mathbf{D}$. While $\mathbf{C}$ does not carry any nonvanishing $L^{1}$-holomorphic quadratic differentials, the disc does. For instance, the norm of the quadratic differential $\tau=d z^{2}$ on the disc is $\pi$.

If $q$ is a quadratic differential at a point $x$ of the disc, we define $\tilde{q}$ to be the holomorphic quadratic differential extending $q$ to $\mathbf{D}$ and minimizing the $L^{1}$-norm. It exists and is unique because of the strict convexity of the $L^{1}$-norm on the space of holomorphic quadratic differentials. One easily checks that $d z^{2}$ is the minimizing holomorphic quadratic differential extending its value at the point 0 . The other minimizing holomorphic quadratic differentials can be deduced from it by using the group of automorphisms of the disc.

Consider the hyperbolic metric

$$
g=\frac{|d z|^{2}}{\left(1-|z|^{2}\right)^{2}}
$$

on the disc, and $T$ an $r$-separated subset of $(\mathbf{D}, g)$, meaning that two distinct points of $T$ are separated by a distance greater than $r$.

Definition 2.1. Let $q=\left\{q_{t}\right\}_{t \in T}$ be a family of quadratic differentials on $T$. We define the Fuchsian series $\sigma(q)$ by

$$
\sigma(q):=\sum_{t \in T} \widetilde{q_{t}} .
$$

The following lemma is the main result of this section, and can be attributed to $\mathrm{H}$. Poincaré $\left[\mathrm{Po}\right.$. We denote $|q|_{\infty, A}$ to be the uniform hyperbolic norm of a quadratic differential $q$ defined on a subset $A \subset \mathbf{D}$.

Lemma 2.2 (Poincaré). If $q$ is bounded in hyperbolic norm, then $\sigma(q)$ converges uniformly on compact subsets of the disc to a holomorphic quadratic differential verifying

$$
|\sigma(q)|_{\infty, \mathbf{D}} \leq C(r)|q|_{\infty, T}
$$

and

$$
|\sigma(q)-q|_{\infty, T} \leq D(r)|q|_{\infty, T},
$$

where $C(r)$ and $D(r)$ are constants depending only on $r$ and $D(r)$ tends to 0 when $r$ goes to infinity.

Proof. First we check some basic properties of the minimizing holomorphic quadratic differentials:

(i) Let $q$ be a quadratic differential at a point $x$ of the disc. The hyperbolic norm of $\tilde{q}$ is a function depending only on the distance to $x$. It can be seen by using the group of isometries of the disc fixing $x$. In particular, if we consider two 
(nonvanishing) quadratic differentials $q_{1}$ and $q_{2}$ at points $x_{1}$ and $x_{2}$ respectively, then we have the symmetry property

$$
\frac{\left|\widetilde{q_{1}}\left(x_{2}\right)\right|}{\left|q_{1}\right|}=\frac{\left|\widetilde{q_{2}}\left(x_{1}\right)\right|}{\left|q_{2}\right|} .
$$

(ii) The hyperbolic norm of an $L^{1}$ quadratic differential $\tau$ on the disc is an integrable function with respect to the hyperbolic volume $v_{g}$ since

$$
\int_{\mathbf{D}}|\tau| d v_{g}=|\tau|_{1}
$$

In particular, using the minimizing quadratic differential $\tau=d z^{2}$ of $d z^{2}$ at the point 0 , we find the general formula

$$
\int_{\mathbf{D}}|\tilde{q}| d v_{g}=\pi|q|
$$

for any quadratic differential $q$ defined at some point $x$.

(iii) By the Cauchy formula, there exists a constant $A(r)$ depending only on $r$ such that

$$
|\tau(0)| \leq A(r) \int_{B_{g}(0, r)}|\tau| d v_{g}
$$

for every holomorphic quadratic differential $\tau$ defined on the hyperbolic ball $B_{g}(0, r)$ of radius $r$ centered at 0 . Again by homogeneity of the hyperbolic disc, we have the uniform inequality

$$
|\tau(x)| \leq A(r) \int_{B_{g}(x, r)}|\tau| d v_{g},
$$

for every holomorphic quadratic differential $\tau$ defined on the hyperbolic ball $B_{g}(x, r)$ of radius $r$ centered at $x$. We choose the function $A$ to be a decreasing function of $r$.

The lemma follows easily from these properties: Let $x \in \mathbf{D}$ and let $q$ be a quadratic differential of norm 1 at $x$. By (i),

$$
\sum_{t \in T}\left|\widetilde{q_{t}}(x)\right| \leq|q|_{\infty, T} \sum_{t \in T}|\tilde{q}(t)|,
$$

and by (iii), we obtain

$$
\sum_{t \in T}\left|\widetilde{q}_{t}(x)\right| \leq|q|_{\infty, T} A(r / 2) \sum_{t \in T} \int_{B_{g}(t, r / 2)}|\tilde{q}| d v_{g} .
$$

Since $T$ is $r$-separated, the balls $B_{g}(t, r / 2)$ are disjoint. Using (ii), we thus have

$$
\sum_{t \in T}\left|\widetilde{q}_{t}(x)\right| \leq C(r)|q|_{\infty, T}
$$

where $C(r)=\pi A(r / 2)$. From this inequality we obtain the uniform convergence of $\sigma(q)$ on compact subsets of the disc.

Now suppose $x$ is a point of $T$. We have

$$
|\sigma(q)(x)-q(x)| \leq \sum_{t \in T, t \neq x}\left|\widetilde{q}_{t}(x)\right|
$$

and doing the same estimates we get

$$
|\sigma(q)(x)-q(x)| \leq A(r / 2)|q|_{\infty, T} \sum_{t \in T, t \neq x} \int_{B_{g}(t, r / 2)}|\tilde{q}| d v_{g} .
$$


Since $T-\{x\}$ is $r$-separated from $x$, we obtain

$$
|\sigma(q)(x)-q(x)| \leq A(r / 2)|q|_{\infty, T} \int_{\mathbf{D}-B_{g}(x, r / 2)}|\tilde{q}| d v_{g} .
$$

But by homogeneity of the disc, $B(R)=\int_{\mathbf{D}-B_{g}(x, R)}|\tilde{q}| d v_{g}$ does not depend on $x$ and because $\tilde{q}$ is integrable it tends to 0 when $R$ goes to infinity. We get Lemma 2.2 with $D(r)=A(r / 2) B(r / 2)$. But because $A$ is decreasing, $D$ tends to 0 when $r$ goes to infinity. This ends the proof of the lemma.

We conclude the section by proving the following extension result.

Proposition 2.3. There exists a constant $r$ such that if $T \subset(\mathbf{D}, g)$ is $r$-separated, every bounded quadratic differential $q$ on $T$ can be extended to the whole disc to a bounded holomorphic one with an estimate

$$
|q|_{\infty, \mathbf{D}} \leq E(r)|q|_{\infty, T} .
$$

Proof. Choose $r$ such that $D(r)<1$. Consider the Banach spaces $Q(T)$ of bounded quadratic differential on $T$ and $H Q(\mathbf{D})$ of bounded holomorphic quadratic differentials on the discs, both equipped with the $L^{\infty}$-norm. If $i: H Q(\mathbf{D}) \rightarrow Q(T)$ is the restriction map and $\sigma: Q(T) \rightarrow H Q(\mathbf{D})$ is the map constructed in Lemma 2.2. then we have

$$
\|i \circ \sigma-i d\| \leq D(r),
$$

where $\|$.$\| is the norm of operator. So because D(r)<1$ the operator $i \circ \sigma$ is invertible and $i$ is thus surjective. The proposition is proved.

\section{A TECHNICAL LEMMA}

Let $r>0$ and $C>0$ be given constants and define $\mathcal{D} \mathcal{Q}$ to be the space of quadratic differentials on the disc, whose hyperbolic norm is bounded everywhere by $C$ and whose support is $r$-separated. In this section we construct a topology on $\mathcal{D} \mathcal{Q}$, and we prove that the map $\sigma$ is continuous with respect to it.

Let $K$ be a compact subset of the disc, $0<\alpha<r / 2$ a real number and $q$ an element of $\mathcal{D} \mathcal{Q}$. Define the subset $U_{K, \alpha, q} \subset \mathcal{D} \mathcal{Q}$ of elements $p$ carrying the following properties:

(iv) For each $t \in \operatorname{supp}(q) \cap K$, there exists at most one element of $\operatorname{supp}(p) \alpha$-close to $t$. If $B_{g}(t, \alpha) \cap \operatorname{supp}(p)$ is empty, then $\left|q_{t}\right|<\alpha$, and if $B_{g}(t, \alpha) \cap \operatorname{supp}(p)=\{s\}$, then $d\left(q_{t}, p_{s}\right)<\alpha$.

(v) For each $s \in \operatorname{supp}(p) \cap K$, there exists at most one element of $\operatorname{supp}(q) \alpha$-close to $s$. If $B_{g}(s, \alpha) \cap \operatorname{supp}(q)$ is empty, then $\left|p_{s}\right|<\alpha$, and if $B_{g}(s, \alpha) \cap \operatorname{supp}(q)=\{t\}$, then $d\left(q_{t}, p_{s}\right)<\alpha$.

The family of sets $U_{K, \alpha, q}$ defines a base of open sets for our topology. The distance $d$ on the total space of the fiber bundle $T^{*} \mathbf{D}^{\otimes 2}$ that we consider has no real significance for our purpose, because $K$ is compact. Thus we will work with the following one:

$$
d\left(q_{1}, q_{2}\right):=\left|\tilde{q_{1}}-\tilde{q_{2}}\right|_{L^{1}},
$$

for all quadratic differentials $q_{1}$ and $q_{2}$ given at some points $x_{1}$ and $x_{2}$ respectively.

Let $B=B_{g}(0,1)$ be the hyperbolic unit ball centered at 0 , and let $H Q(B)$ be the space of holomorphic quadratic differentials on $B$ with bounded hyperbolic norm. We equip $H Q(B)$ with the uniform topology.

Lemma 3.1. The map $\sigma: \mathcal{D Q} \rightarrow H Q(B)$ is continuous. 
Proof. Let $q \in \mathcal{D} \mathcal{Q}, R>r$ and $0<\alpha<r / 2$ be real numbers. Now choose $p$ in the set $U_{\bar{B}_{g}(0, R), \alpha, q}$. We will show that $|\sigma(p)-\sigma(q)|_{\infty, B}$ tends to 0 uniformly when $R$ tends to infinity and when $\alpha=o\left(1 / \operatorname{vol}\left(B_{g}(0, R)\right)\right)$. This suffices to prove the lemma.

Define $T$ to be in $\bar{B}_{g}(0, R)$ the union of the points of $\operatorname{supp}(q)$ and of the points of $\operatorname{supp}(p)$ from which there is no $\alpha$-close point of $\operatorname{supp}(q)$. Similarly, define $S$ to be in $\bar{B}_{g}(0, R+\alpha)$ the union of the points of $\operatorname{supp}(p)$ and of the points of $\operatorname{supp}(q)$ from which there is no $\alpha$-close point of $\operatorname{supp}(p)$. By (iv) we can thus associate to every point $t$ of $T$ a unique point $s(t)$ of $S \cap B_{g}(t, \alpha)$. The map $s: T \rightarrow S$ is injective and by (v) its image contains $S \cap \bar{B}_{g}(0, R-\alpha)$. We thus have, for each $x$ in the unit hyperbolic ball $B$,

$$
\begin{aligned}
|\sigma(q)(x)-\sigma(p)(x)| \leq \sum_{t \in T} & \left|\widetilde{q_{t}}(x)-\widetilde{p_{s(t)}}(x)\right| \\
& +\sum_{t \in \operatorname{supp}(q)-B_{g}(0, R)}\left|\widetilde{q_{t}}(x)\right|+\sum_{s \in \operatorname{supp}(p)-B_{g}(0, R-\alpha)}\left|{\widetilde{p_{s}}}_{s}(x)\right| .
\end{aligned}
$$

We first bound the first term of the right-hand side of (3.1). By definition of the map $t \in T \mapsto s(t) \in S$, and by property (iv),

$$
\left|\widetilde{q_{t}}-\widetilde{p_{s(t)}}\right|_{1}<\alpha,
$$

so that using the uniform Gårding inequality (iii), we find

$$
\left|\widetilde{q_{t}}(x)-\widetilde{p_{s(t)}}(x)\right| \leq A(\infty) \alpha .
$$

In order to bound on the number $|T|$ of points of $T$, observe that $T$ is a priori only an $\alpha$-separated subset, but by definition it is the union of two $r$-separated subsets of the ball $B_{g}(0, R)$. Thus we obtain

$$
\sum_{t \in T}\left|\widetilde{q}_{t}(x)-\widetilde{p_{s(t)}}(x)\right| \leq A(\infty) \alpha|T| \leq 2 A(\infty) \alpha \frac{\operatorname{vol}\left(B_{g}(0, R+r / 2)\right)}{\operatorname{vol}\left(B_{g}(0, r / 2)\right)} .
$$

For the other terms of (3.1), we proceed as in Lemma 2.2 and we get

$$
\sum_{t \in \operatorname{supp}(q)-B_{g}(0, R)}\left|\widetilde{q}_{t}(x)\right| \leq A(r / 2) B_{g}(R-\alpha-1)|q|_{\infty}
$$

and

$$
\sum_{s \in \operatorname{supp}(p)-B_{g}(0, R-\alpha)}\left|\widetilde{p_{s}}(x)\right| \leq A(r / 2) B_{g}(R-2 \alpha-1)|q|_{\infty} .
$$

Recall that the elements of $\mathcal{D} \mathcal{Q}$ are supposed to be bounded by a universal constant $C>0$, so we conclude that

$$
|\sigma(q)-\sigma(p)|_{\infty, B} \leq F\left(o(R)+\alpha \operatorname{vol}\left(B_{g}(0, R)\right)\right),
$$

for a constant $F$ depending only on $r$ and $C$. The lemma is proved.

\section{Continuity of Laminated fuchsian Series}

Let $\mathcal{L}$ be a Riemann surfaces lamination of a topological space $X$.

Definition 4.1. A transversal of $\mathcal{L}$ is a closed subset $\mathcal{T} \subset X$ satisfying the following property: There exists a cover of $X$ by flow boxes $\mathbf{D} \times T$ such that $\mathcal{T}$ intersects each slice $\mathbf{D} \times\{t\}$ in at most one point. 
On a transversal there is the natural transverse topology. For instance, a point $t$ of $\mathcal{T}$ is a boundary point (resp. interior point) if there exists a flow box $\mathbf{D} \times T$ containing $t$ and such that $t$ projects on a boundary point (resp. interior point) of the image of $\mathcal{T}$ by the projection $\mathbf{D} \times T \rightarrow T$. The boundary of $\mathcal{T}$ in the transverse topology is denoted by $\partial_{t} \mathcal{T}$, and the interior $\operatorname{Int}_{t}(T)$.

Suppose now that the leaves of $\mathcal{L}$ are hyperbolic and the space $X$ is compact. A. Candel Ca and A. Verjovsky Ve have shown that the hyperbolic metric on the leaves is continuous. Thus every leaf with its hyperbolic metric intersects a transversal $\mathcal{T}$ in an $r$-separated subset, where $r=r(\mathcal{T})$ is a positive number depending only on $\mathcal{T}$. If $q$ is a bounded quadratic differential on $\mathcal{T}$, we consider on the universal cover $\pi: \widetilde{L} \rightarrow L$ of each leaf the series

$$
\sigma(q)=\sum_{t \in \pi^{-1}(L \cap \mathcal{T})} \widetilde{q_{\pi(t)}} .
$$

Because this series converges (Lemma 2.2) and is invariant by the fundamental group of $L$, it induces a holomorphic quadratic differential the leaves of $\mathcal{L}$. Here is the main result of the section.

Lemma 4.2. Suppose $q$ is a continuous quadratic differential defined on a transversal $\mathcal{T}$ and vanishing on $\partial_{t} \mathcal{T}$. Then $\sigma(q)$ is continuous.

Proof. To prove this lemma, we will use the uniformization theory of A. Candel and A. Verjovsky for hyperbolic laminations of a compact space. Take a point $x \in X$ and a transversal $T$ containing $x$ in its interior for the transverse topology. If we choose a continuous family $v_{t}$ of unitary vectors of the unitary tangent bundle $U \mathcal{L}$ of $\mathcal{L}$ on $T$, then we can consider the map

$$
\pi: \mathbf{D} \times T \rightarrow X
$$

defined on each plaque $\mathbf{D} \times\{t\}$ as being the covering sending $(0, t)$ to $t$ and the vector $\frac{\partial}{\partial x} \in T_{0} \mathbf{D}$ of the unit disc to $v_{t}$. The uniformization result of A. Candel and A. Verjovsky $\left[\mathrm{Ca}, \mathrm{Ve}\right.$ is the fact that this map induces by restriction to $\mathbf{D} \times \operatorname{Int}_{t}(T)$ a local biholomorphism of Riemann surfaces laminations. In particular, if $\varepsilon>0$ is chosen small enough, the restriction of $\pi$ to $B_{g}(0, \varepsilon) \times \operatorname{Int}_{t}(T)$, where $B_{g}(0, \varepsilon)$ is the hyperbolic ball of radius $\varepsilon$, defines a coordinate in which we will prove that $\sigma(q)$ is continuous and the lemma will follow.

First, the preimage $\hat{\mathcal{T}}$ of $\mathcal{T}$ by $\pi$ is a transversal of the trivial lamination $\mathbf{D} \times T$, and the differential $\pi^{*} q$ is continuous on it and vanishes on $\partial_{t} \hat{\mathcal{T}}$. Furthermore, $q$ is bounded (say by $C$ ), and the intersection of $\mathcal{T}$ with each leaf is $r$-separated, so that the family of quadratic differentials $\pi^{*} q_{\mid \mathbf{D} \times\{t\}}$ defines a map from $T$ to the space $\mathcal{D} Q$ that we have considered in the Section 3. By Lemma 3.1 it suffices to prove that it is continuous.

Take $t_{0} \in \operatorname{Int}_{t}(T)$, and let $R>r, 0<\alpha<r / 2$ be two real numbers. The ball $\bar{B}_{g}(0, R) \times\left\{t_{0}\right\}$ intersects $\hat{\mathcal{T}}$ in a finite number of points, say $\left(z_{i}, t_{0}\right), i=1, \ldots, N$. Let $T_{i}$ be a neighborhood of $t_{0}$ in $T$ such that the flow box $U_{i}=B_{g}\left(z_{i}, \alpha\right) \times T_{i}$ contains at most one point of $\hat{\mathcal{T}}$ in every plaque $B_{g}\left(z_{i}, \alpha\right) \times\{t\}$. Since $\pi^{*} q$ is continuous on $\hat{\mathcal{T}}$, we can also choose $T_{i}$ in such a way that if $(z, t)$ and $\left(z^{\prime}, t^{\prime}\right)$ are two points of $\hat{\mathcal{T}} \cap U_{i}$, then

$$
d\left(\pi^{*} q_{(z, t)}, \pi^{*} q_{\left(z^{\prime}, t^{\prime}\right)}\right)<\alpha .
$$


Consider now $\mathcal{V}=T_{1} \cap \ldots \cap T_{N}$. The construction of the $T_{i}$ 's and the vanishing of $\pi^{*} q$ on $\partial_{t} \hat{\mathcal{T}}$ is exactly the same as saying that for each $t \in \mathcal{V}, \pi^{*} q_{\mid \mathbf{D} \times\{t\}} \in \mathcal{U}_{\bar{B}_{g}(0, R), \alpha, q_{0}}$, $q_{0}$ being the quadratic differential $\pi^{*} q_{\mid \mathbf{D} \times\left\{t_{0}\right\}}$. Thus we have proved continuity of the map $t \in T \mapsto \pi^{*} q_{\mid \mathbf{D} \times\{t\}} \in \mathcal{D} \mathcal{Q}$ and therefore the continuity of $\sigma(q)$ by using Lemma 3.1 .

\section{Proof of Theorem 1.1}

The proof of Theorem 1.1 is a variation of the open image theorem that we used in Proposition 2.3. If $L$ is a simply connected leaf of $\mathcal{L}$, there exists a transversal $T_{1}$ cutting $L$ in an interior point $t_{1}$ of $T_{1}$ with $r=r\left(T_{1}\right)$ arbitrarily large such that $D(r)<1$. We can suppose furthermore that the bundle $T^{*} \mathcal{L}^{\otimes 2}$ is trivial on $T_{1}$ if, for instance, $T_{1}$ is chosen in a flow box.

First suppose that $L$ is not transversally isolated (i.e., $t_{1}$ is not isolated in $T_{1}$ ), and take a closed neighborhood $T_{2}$ of $t_{1}$ in $T_{1}$. We will show that every continuous quadratic differential on $T_{2}$ can be extended to $X$ to a continuous holomorphic one, and this will prove the result because $T_{2}$ is infinite.

We begin by extending $q$ to a continuous quadratic differential $\bar{q}$ on $T_{1}$ vanishing on $\partial_{t} T_{1}$ and such that $|\bar{q}|_{T_{1}, \infty} \leq|q|_{T_{2}, \infty}$. Then we consider

$$
\sigma_{1}=\sigma(\bar{q})
$$

By Lemma 4.2 $\sigma_{1}$ is a continuous holomorphic quadratic differential on $X$ such that

$$
\left|\sigma_{1}\right|_{X, \infty} \leq C(r)|q|_{\infty, T_{2}}
$$

and

$$
\left|q-\left(\sigma_{1}\right)_{\mid T_{2}}\right|_{\infty, T_{2}} \leq D(r)|q|_{\infty, T_{2}} .
$$

This is the first step of an approximation process that goes inductively as follows. Given continuous holomorphic quadratic differentials $\sigma_{1}, \ldots, \sigma_{n}$ on $X$ satisfying for $i=1, \ldots, n-1$,

$$
\left|\sigma_{i+1}\right|_{X, \infty} \leq C(r)\left|q-\left(\sigma_{1}+\ldots+\sigma_{i}\right)_{\mid T_{2}}\right|_{\infty, T_{2}}
$$

and

$$
\left|q-\left(\sigma_{1}+\ldots+\sigma_{i+1}\right)_{\mid T_{2}}\right|_{\infty, T_{2}} \leq D(r)\left|q-\left(\sigma_{1}+\ldots+\sigma_{i}\right)_{\mid T_{2}}\right|_{\infty, T_{2}},
$$

we construct $\sigma_{n+1}$ by considering $\sigma_{n+1}=\sigma_{1}\left(q-\left(\sigma_{1}+\ldots+\sigma_{n}\right)_{T_{2}}\right)$ rather than $q$ (see (5.1)). By construction both (5.2) and (5.3) are also verified for $i=n$. Thus we have for each $n \geq 1$,

$$
\left|q-\left(\sigma_{1}+\ldots+\sigma_{n}\right)_{\mid T_{2}}\right| \leq D(r)^{n}|q|_{\infty, T_{2}}
$$

and

$$
\left|\sigma_{n}\right|_{\infty, X} \leq C(r) D(r)^{n-1}|q|_{\infty, T_{2}} .
$$

The series $\sum_{n} \sigma_{n}$ converges uniformly to a continuous holomorphic quadratic differential on $X$ whose restriction to $T_{2}$ is $q$. The proof of the theorem in that case is achieved.

Remark 5.1. In fact, we have thus proved the theorem under the weaker hypothesis that the hyperbolic Riemann surface lamination has a leaf $L$ and a point $x$ on $L$ such that $L$ is not transversally isolated and the radius $r$ of injectivity at $x$ for the hyperbolic metric in $L$ verifies $D(r)<1$. 
Now, if there exists on $\mathcal{L}$ a simply connected leaf $L$ which is transversally isolated, then every holomorphic quadratic differential on $L$ whose hyperbolic norm tends to 0 at infinity can be extended by 0 to a continuous holomorphic quadratic differential on $X$. So the space of continuous holomorphic quadratic differentials contains at least the one defined on $L$ by the formula

$$
\tau=f(z) d z^{2},
$$

where $z: L \rightarrow \mathbf{D}$ is a biholomorphism and $f$ is a holomorphic function on the disc bounded at infinity. This completes the proof of the theorem.

\section{REFERENCES}

[Ca] A. Candel. Uniformization of surface laminations. Ann. Sci. École Norm. Sup.(4) 26 (1993), no.4, pp. 489-516. MR.1235439 (94f:57025)

[Gh] É. Ghys. Laminations par surfaces de Riemann. Panor. Synthèses 8 (1999), pp. 49-95. MR:1760843 (2001g:37068)

[Po] H. Poincaré. Sur les fonctions fuchsiennes. Acta Math. 1 (1882), pp. 193-294.

[Su] D. Sullivan. Linking the universalities of Milnor-Thurston, Feigenbaum and Ahlfors-Bers. Topological methods in modern mathematics, (Stony Brook, NY, 1991), pp. 543-564. MR:1215976 (94c:58060)

[Ve] A. VerJovSKY. A uniformization theorem for holomorphic foliations. The Lefschetz centennial conference, Contemp. Math., vol. 58, 1987, pp. 233-253. MR0893869 (88h:57027)

Laboratoire de Mathématiques, Université Paris-Sud-BÂt 425, 91405 Orsay Cedex, CNRS UMR 8628, FRANCE

E-mail address: Bertrand.Deroin@math.u-psud.fr 